\title{
Appropriateness of the request for laboratory tests in the diagnosis of systemic autoimmune diseases
}

\author{
Roberta Marotta', Rossana Baccalini'², Wanda Patrizia Porreca², Gianlodovico Melzi d'Eril' \\ I Dipartimento Di Scienze della Salute, Università degli Studi di Milano \\ 2 Laboratorio analisi chimico cliniche e microbiologiche, A.O. San Paolo, Milano
}

Key words: Appropriateness, Laboratory tests, Autoimmunity

\section{Appropriatezza della richiesta di esami di laboratorio nella diagnosi delle malattie autoimmuni sistemiche}

\section{SUMMARY}

Autoimmune diseases are caused by an immune response against some autoantigens.

This response, characterized by the presence of autoantibodies, causes an inflammatory disease, which can cause a damage of specific organ (organ-specific autoimmune diseases) or systemic (systemic autoimmune diseases). The presence of these autoantibodies in the serum can therefore be a useful parameter for the diagnosis and/or monitoring of autoimmune diseases

In this paper we describe a flow chart about laboratory tests in the diagnosis of systemic autoimmune diseases so as to maintain appropriateness of the request and contain costs.

\section{INTRODUZIONE}

Le malattie autoimmuni sono un gruppo eterogeneo di patologie caratterizzate da una risposta immune diretta verso costituenti propri dell'organismo (autoantigeni) (1).

Questa risposta, caratterizzata dalla presenza di autoanticorpi, provoca una patologia infiammatoria che può causare un danno d'organo specifico (malattie autoimmuni organo-specifiche) o sistemico (malattie autoimmuni sistemiche).

La presenza di tali autoanticorpi nel siero può essere dunque un parametro utile per la diagnosi e/o il monitoraggio delle malattie autoimmuni, tuttavia il loro significato non è sempre di facile interpretazione.

Ad oggi, i test commerciali a disposizione per lo studio delle malattie autoimmuni, sono numerosi e diversificati: ai classici metodi di immunofluorescenza indiretta (IFI), si sono aggiunti metodi immunoenzimatici (ELISA), immunoblotting e arrays antigenici nel tentativo di migliorare la sensibilità e specificità analitica. Tale eterogeneità costituisce un vantaggio in quanto permette di utilizzare più metodi in associazione e migliorare l'indagine diagnostica, ma contemporaneamente è necessario mantenere 1'appropriatezza della richiesta e contenere la spesa economico-sanitaria.

Con tale obiettivo, vari gruppi di lavoro nell'ambito della comunità scientifica a livello nazionale e internazionale, hanno elaborato linee guida (3) per il corretto utilizzo dei marcatori autoanticorpali. Nelle diverse proposte, di fronte a segni e sintomi suggestivi di una specifica patologia autoimmune, è consigliata la ricerca degli anticorpi anti-nucleo (ANA) come test di primo livello maggiormente sensibile ed economico. La bassa specificità impone, però, un'accurata selezione dei pazienti.

Solo in caso di reattività vengono eseguite diluizioni seriate al fine di definire il titolo e il pattern anticorpale (2). Si deve ricorrere alla determinazione degli anticorpi anti-antigeni estraibili (ENA), quindi ad un test più specifico di secondo livello, quando la positività ANA è uguale o superiore a 1:160 o in assenza di positività ANA, qualora vi siano sintomi clinici. Inutile l'esecuzione di un test ENA screening in presenza di un quadro fluoroscopio dove la specificità anticorpale è facilmente desumibile (pattern centromerico) o non identificabile tramite un classico test ENA a 6-8 antigeni (pattern nuclear dots).

Solo nel caso di positività del test ENA screening si procede all'esecuzione di un immunoblotting per la determinazione dello specifico antigene.

In tutti i casi in cui il test ANA in fluorescenza risulti positivo al titolo uguale o superiore a 1:160 con pattern omogeneo è raccomandata la determinazione di anticorpi anti-DNA nativo a doppia elica (dsDNA).

\section{MATERIALI E METODI}

Il metodo raccomandato dalle diverse linee guida per la ricerca degli ANA è l'immunofluorescenza indiretta (IFI), in quanto permette di stabilire sia il titolo sia il pattern fluoroscopico, oltre ad essere

\section{Corresponding author: Roberta Marotta}

Via A. Di Rudiní 8 - 20142 Milano

Tel.: 0281844237 - Fax: 0281844027

E-mail: roberta.marotta@ao-sanpaolo.it; roberta.marotta@studenti.unimi.it 
una tecnica a basso costo e di facile esecuzione. Come substrato, viene raccomandato l'impiego di cellule epiteliali da carcinoma laringeo umano (HEp-2, American Type Culture Collection CCL 23), preferibili ad altri tipi di substrato (fegato di topo e/o ratto) in quanto costituito da popolazioni cellulari omogenee asincrone di origine umana, con nuclei e nucleoli evidenti ed elevato indice mitotico in grado di evidenziare anche gli antigeni presenti solo in determinate fasi del ciclo cellulare. Il titolo consigliato per effettuare lo screening è $1 / 80$.

Per lo screening ANA sono presenti in commercio anche prodotti diagnostici basati su metodo immunoenzimatico ELISA (dosaggio qualitativo), tuttavia essi possono solo affiancare ma non sostituire il metodo IFI, in quanto non sempre in grado di esprimere tutti gli antigeni necessari per la determinazione degli ANA.

Per la ricerca degli anticorpi anti-dsDNA possono essere utilizzati sia il metodo IFI su Crithidia luciliae (emoflagellato con chinetoplasto che contiene dsDNA allo stato nativo) sia il metodo ELISA. In questo caso è opportuno confermare le positività con un metodo più specifico (IFI); se invece si utilizza per primo il metodo IFI è necessario che $\mathrm{i}$ risultati positivi vengano poi espressi in termini quantitativi ritestando i campioni con un metodo immunoenzimatico calibrato sullo standard internazionale WHO/Wo80.

Per quanto riguarda gli anti-ENA, il test può essere eseguito con uno dei vari metodi disponibili in commercio, scelto in base alle esigenze del proprio laboratorio. Tuttavia nessuno di essi ha una sensibilità del $100 \%$, quindi in presenza di un quadro clinico caratteristico e dopo il riscontro di una positività ad alto titolo degli ANA, un'eventuale negatività degli anti-ENA deve essere confermata con almeno due metodi diversi.

Presso il Laboratorio Analisi dell'A.O. San Paolo di Milano la ricerca degli ANA e degli anticorpi anti-dsDNA viene effettuata rispettivamente su cellule Hep2 e su Crithidia luciliae mediante metodo di immunofluorescenza indiretta (Euroimmun).

La ricerca degli ENA viene eseguita mediante metodo immunoenzimatico ELISA (Euroimmun) e ogni seduta viene valutata verso controlli positivi e negativi a titolo noto (Biorad Liquicheck, Italia).

\section{RISULTATI}

Presso l'A.O. San Paolo negli anni 2010, 2011 e nei primi 5 mesi del 2012 le richieste interne per il dosaggio degli ANA sono state rispettivamente 553,627 e 238 rispetto ad un totale di accettazioni pari a 1639,1800 e 742 . Il 48\% delle richieste interne è stato effettuato dai reparti di medicina, ''11\% dalla pediatria, il 6\% dalla neurologia e dermatologia, il $3 \%$ dalla nefrologia e il restante $28 \%$ da altri reparti. Nei tre periodi indicati, nel $27 \%$ dei casi è stato evidenziato un primo riscontro di reattività, per la quale si sarebbe resa necessaria la ricerca degli ENA. Tuttavia le richieste per tale dosaggio nei medesimi anni sono state rispettivamente di 378 (68\%), $393(62.7 \%)$ e 140 (59\%). Campioni reattivi con titolo uguale o maggiore 1:160 e pattern omogeneo sono stati 15 negli anni $2010(2.7 \%)$ e $2011(2.4 \%)$ e 5 fino a maggio $2012(2.1 \%)$. Nei medesimi periodi le richieste pervenute al laboratorio per la ricerca degli anticorpi anti-dsDNA sono state rispettivamente 247 (44.6\%), 209 (33.3) e 79 (33.1\%).

\section{CONCLUSIONI}

La ricerca di anticorpi anti-nucleo è il primo test da eseguire in caso di sospetto clinico di MAIS. Solo in caso di positività significativa sarebbe utile procedere con approfondimenti diagnostici. Allo scopo di migliorare l'appropriatezza delle richieste provenienti dai reparti dell'A.O. San Paolo, è stato elaborato un referto interpretativo approvato dalla Direzione Sanitaria che permetta al clinico di rispettare il percorso diagnostico previsto dalle linee guida in materia di malattie autoimmuni.

In tal modo oltre a raggiungere un'appropriatezza della richiesta, si otterrebbe un'ottimizzazione dal punto di vista delle risorse economiche ed umane. Infatti, secondo il tariffario di Regione Lombardia, il costo totale delle prestazioni svolte in routine per ENA è stato rispettivamente per gli anni 2010, 2011 e per i primi 5 mesi del 2012 di € 5190, $€ 5400$ e $€ 1920$ ovvero circa il $60 \%$ in più per ogni anno rispetto al costo associato ad una richiesta congruente. La medesima condizione si evidenzia per il test nDNA, per la quale un'accettazione appropriata fruirebbe un risparmio economico di circa il $90 \%$.

Obiettivo dell'U.O. Laboratorio Analisi per il secondo semestre dell'anno 2012 è quello di perseguire una migliore appropriatezza della richiesta per la diagnostica MAIS.

\section{BIBLIOGRAFIA}

1. Betterle C. Le malattie autoimmuni, Piccin editore, 2000

2. Bizzaro N. L'appropriatezza nella richiesta dei test autoanticorpali per la diagnosi delle malattie reumatiche autoimmuni. Med Lab-JLM 2001;2:11-16

3. XL Congresso Nazionale AMCLI (2011): Percorso diagnostico per la ricerca di autoanticorpi nelle connettiviti. 